\title{
Analytical Treatment of Volterra Integro-Differential Equations of Fractional Derivatives
}

\author{
*Ghasemi M.; Department of Applied Mathematics, Faculty of \\ Mathematical Sciences, Shahrekord University, Shahrekord, Iran. \\ Babolian E.; Faculty of Mathematical Sciences and Computer, \\ Kharazmi University
}

Received: 18 Nov 2013

Revised: 10 Nov 2014

\begin{abstract}
In this paper the solution of the Volterra integro-differential equations of fractional order is presented. The proposed method consists in constructing the functional series, sum of which determines the function giving the solution of considered problem. We derive conditions under which the solution series, constructed by the method is convergent. Some examples are presented to verify convergence, efficiency and simplicity of the method.

Mathematics Subject Classification: 45J05, 65T60
\end{abstract}

Keywords: Fractional Volterra integro-differential equations, Caputo fractional derivative, Riemann-Liouville fractional derivative.

\section{Introduction}

In recent years, various kinds of analytical and numerical methods were used to solve fractional integro-differential equations. Rawashdeh [1] applied collocation method to study fractional integro-differential equations. Authors of [2] applied the Adomian decomposition method (ADM) to approximate solutions for fourth-order fractional integro-differential equations. The Haar wavelet method were used to solve the fractional integral equations [3]. Applied fractional differential transform method was employed to approximate solutions for fractional integro-differential equations in [4]. The homotopy analysis method for solving the fractional Volterra's population system has been presented in [5]. A recent application has included numerically determining solutions for various classes of nonlinear fractional equations in $[6,7,8]$. In this paper,

\footnotetext{
*Corresponding author: meh_ghasemi@yahoo.com
} 
we apply variational iteration method (VIM) $[9,10,11,12,13,14,15]$ to solve Volterra integro-differential equations. This method is widely used by many researchers to study linear and nonlinear problems. This method is employed in [16] to solve the KleinGordon partial differential equations. Authors of [17] applied the variational iteration method to solve the Lane-Emden differential equation. For more applications of the method, we refere to $[18,19,20,21]$.

In this study, we consider Volterra integro-differential equations (VI-DEs) of fractional order of the form

$$
\left\{\begin{array}{c}
{ }^{c} D_{0_{+}}^{\alpha} \zeta(x)-\lambda \int_{0}^{x} k(x, t) \zeta(\mathrm{t}) \mathrm{dt}=\mathrm{g}(x), \\
\zeta^{(\mathrm{i})}(0)=\mathrm{c}_{\mathrm{i}}, \mathrm{i}=0,1,2, \ldots, \mathrm{n}-1, \mathrm{n}=[\alpha]+1 .
\end{array}\right.
$$

where $g \in L^{2}([0, X]), k$ (separable $) \in L^{2}\left([0, X]^{2}\right)$ are given functions, ${ }^{c} D_{0_{+}}^{\alpha}$ is the fractional derivative, and $\zeta(\mathrm{x})$ is unknown function. The paper is organized as follows: In Section 2, we recall some basic definitions and properties of the fractional calculus theory. In Section 3, we construct an algorithm for solving Volterra integro-differential equations of fractional order by using the VIM. In Section 4, we derive the convergence conditions for the VIM to solve the Volterra integro-differential equations of fractional order. In Section 5, some illustrative examples are given. Some concluding remarks are given in Section 6.

\section{Preliminaries}

We now present the definitions and auxiliary results for fractional calculus which are used in this paper. For more details on the mathematical properties of fractional derivatives and integrals see $([22,23])$.

Definition 2.1. ([22]) The fractional integral of order $\alpha \geq 0$ of a function $\zeta(x)$ : $(0, \infty) \rightarrow R$ is defined as

$$
\begin{cases}I_{0_{+}}^{\alpha} \zeta(x)=\frac{1}{\Gamma(\alpha)} \int_{0}^{x}(x-s)^{(\alpha-1)} \zeta(s) \mathrm{d} s, & \alpha>0, \\ I_{0_{+}}^{0} \zeta(x)=\zeta(x), & \end{cases}
$$

where $\Gamma$ is the well-known Gamma function.

Definition 2.2 ([22]). The fractional derivative of order $\alpha \geq 0$ of a continuous function $\zeta(x):(0, \infty) \rightarrow R$ is defined as

$$
{ }^{c} D_{0_{+}}^{\alpha} \zeta(x)=\frac{1}{\Gamma(\mathrm{n}-\alpha)} \int_{0}^{x}(x-s)^{\mathrm{n}-\alpha-1} \zeta^{(\mathrm{n})}(s) \mathrm{ds}, \mathrm{n}=[\alpha]+1 .
$$


where $[\alpha]$ is the integer part of $\alpha$.

Definition 2.3 ([23]). The Mittag-Leffler function $E_{\alpha, \beta}(z)$ with $\alpha>0, \beta>0$ is defined by the following series representation, which is valid in the whole complex plane

$$
E_{\alpha, \beta}(z)=\sum_{n=0}^{\infty} \frac{z^{n}}{\Gamma(n \alpha+\beta)}
$$

Lemma 2.1 ([22]). Riemann-Liouville factional integral and derivative have the following properties
(i) $I_{0_{+}}^{\alpha} \zeta(x) I_{0_{+}}^{\beta} \zeta(x)=I_{0_{+}}^{\alpha+\beta} \zeta(x)$,
(ii) $I_{0_{+}}^{\alpha} \zeta(x) I_{0_{+}}^{\beta} \zeta(x)=I_{0_{+}}^{\alpha} \zeta(x) I_{0_{+}}^{\beta} \zeta(x)$,
(iii) $I_{0_{+}}^{\alpha} x^{\gamma}=\frac{\Gamma(\gamma+1)}{\Gamma(\alpha+\gamma+1)} x^{\alpha+\gamma}$
(iv) $I_{0_{+}}^{\alpha}{ }^{c} D_{0_{+}}^{\alpha} \zeta(x)=\zeta(x)-\sum_{\mathrm{k}=0}^{\mathrm{n}-1} \zeta^{(\mathrm{k})}\left(0^{+}\right) \frac{x^{\mathrm{k}}}{\mathrm{k} !}$
(v) ${ }^{c} D_{0_{+}}^{\alpha} I_{0_{+}}^{\alpha} \zeta(x)=\zeta(x)$.

\section{Main Results}

Consider the following Volterra integral equation where ${ }^{c} D_{0_{+}}^{\alpha}$ is the fractional derivative:

$$
\left\{\begin{array}{l}
{ }^{c} D_{0_{+}}^{\alpha} \zeta(x)-\lambda \int_{0}^{x} k(x, t) \zeta(\mathrm{t}) \mathrm{dt}=\mathrm{g}(x) \\
\zeta^{(\mathrm{i})}(0)=\mathrm{c}_{\mathrm{i}}, \mathrm{i}=0,1,2, \ldots, \mathrm{n}-1, \mathrm{n}=[\alpha]+1 .
\end{array}\right.
$$

Theorem.3.1. The general solution of the Eq. (1.1) is equivalent to the mixed Volterra integral equation in the following form:

$$
\zeta(x)=I_{0_{+}}^{\alpha}(g(x))+\sum_{j=0}^{n-1} \mathrm{c}_{\mathrm{j}} \frac{x^{\mathrm{j}}}{\mathrm{j} !}+\lambda \frac{1}{\Gamma(\alpha)} \int_{0}^{x} \int_{0}^{s}(x-s)^{(\alpha-1)} k(x, t) \zeta(\mathrm{t}) \mathrm{dtd} .
$$

Proof. Applying the operator $I_{0_{+}}^{\alpha}$, the inverse of the operator $D_{0_{+}}^{\alpha}$, to both sides of Eq. (3.1) yields:

$$
\zeta(x)=I_{0_{+}}^{\alpha}(g(x))+\sum_{j=0}^{n-1} c_{\mathrm{j}} \frac{x^{\mathrm{j}}}{\mathrm{j} !}+\lambda I_{0_{+}}^{\alpha}\left(\int_{0}^{x} k(x, t) \zeta(\mathrm{t}) \mathrm{dt}\right)
$$

which completes the proof.

\subsection{Iterative method}

Now we consider the VI-DEs as a mixed Volterra integral equation, which reads as: 


$$
\zeta(x)=I_{0_{+}}^{\alpha}(g(x))+\sum_{\mathrm{j}=0}^{\mathrm{n}-1} \mathrm{c}_{\mathrm{j}} \frac{x^{\mathrm{j}}}{\mathrm{j} !}+\lambda I_{0_{+}}^{\alpha}\left(\int_{0}^{x} k(x, t) \zeta(\mathrm{t}) \mathrm{dt}\right) .
$$

There is a simple iteration formula for (3.2) in the form:

$$
\zeta_{\mathrm{k}}(x)=I_{0_{+}}^{\alpha}(g(x))+\sum_{\mathrm{j}=0}^{\mathrm{n}-1} \mathrm{c}_{\mathrm{j}} \frac{x^{\mathrm{j}}}{\mathrm{j} !}+\lambda I_{0_{+}}^{\alpha}\left(\int_{0}^{x} k(x, t) \zeta_{\mathrm{k}-1} \mathrm{dt}\right), \mathrm{k}=1,2, \ldots
$$

Beginning with $\zeta_{0}(x)=I_{0_{+}}^{\alpha}(g(x))+\sum_{\mathrm{j}=0}^{\mathrm{n}-1} \mathrm{c}_{\mathrm{j}} \frac{x^{\mathrm{j}}}{\mathrm{j} !}$, the approximate solution of Eq. (1.1) can be determined by the iterative formula Eq. (3.3).

\subsection{Stability of the solution}

We now investigate the changes in the obtained solution which are caused by small perturbations in the initial conditions.

Theorem.3.2 Let $\zeta(\mathrm{x})$ and $\tilde{\zeta}(x)$ be the solutions of the following problems:

$$
\left\{\begin{array}{c}
{ }^{c} D_{0_{+}}^{\alpha} \zeta(x)-\lambda \int_{0}^{x} k(x, t) \zeta(\mathrm{t}) \mathrm{dt}=\mathrm{g}(x), \\
\zeta^{(\mathrm{i})}(0)=\mathrm{c}_{\mathrm{i}}, \mathrm{i}=0,1,2, \ldots, \mathrm{n}-1, \quad \mathrm{n}-1<\alpha \leq n,
\end{array}\right.
$$

and

$$
\left\{\begin{array}{l}
{ }^{c} D_{0_{+}}^{\alpha} \zeta(x)-\lambda \int_{0}^{x} k(x, t) \zeta(\mathrm{t}) \mathrm{dt}=\mathrm{g}(x), \\
\zeta^{(\mathrm{i})}(0)=\mathrm{b}_{\mathrm{i}}, \mathrm{i}=0,1,2, \ldots, \mathrm{n}-1, \quad \mathrm{n}-1<\alpha \leq n,
\end{array}\right.
$$

where $\left|b_{i}-c_{i}\right| \leq \epsilon_{i}, i=0,1,2, \ldots, n-1$ and suppose that there exists a constant $N$ such that:

$$
|k(x, t)| \leq N, \quad \forall(x, t) \in[0, X]^{2},
$$

then we have

$$
|\zeta(x)-\tilde{\zeta}(x)| \leq \sum_{\mathrm{j}=0}^{\mathrm{n}-1}\left|\epsilon_{\mathrm{j}}\right| x^{\mathrm{j}} \mathrm{E}_{\delta+1, \mathrm{j}+1}\left(|\lambda| N x^{\delta+1}\right) .
$$

Proof. In accordance with the previous subsection, we have:

$$
\begin{gathered}
\zeta(x)=\lim _{k \rightarrow \infty} \zeta_{k}(x), \\
\zeta_{0}(x)=I_{0_{+}}^{\alpha}(g(x))+\sum_{\mathrm{j}=0}^{\mathrm{n}-1} \mathrm{c}_{\mathrm{j}} \frac{x^{\mathrm{j}} \mathrm{j} !}{x} \\
\zeta_{\mathrm{k}}(x)=\zeta_{0}(x)+\lambda I_{0_{+}}^{\alpha}\left(\int_{0}^{\alpha} k(x, t) \zeta_{\mathrm{k}-1} \mathrm{dt}\right), \mathrm{k}=1,2, \ldots
\end{gathered}
$$

and

$$
\begin{gathered}
\tilde{\zeta}(x)=\lim _{k \rightarrow \infty} \tilde{\zeta}_{k}, \\
\tilde{\zeta}_{0}(x)=I_{0_{+}}^{\delta}(g(x))+\sum_{\mathrm{j}=0}^{\mathrm{n}-1} \mathrm{~b}_{\mathrm{j}} \frac{x^{\mathrm{j}}}{\mathrm{j} !}
\end{gathered}
$$




$$
\tilde{\zeta}_{\mathrm{k}}(x)=\tilde{\zeta}_{0}(x)+\lambda I_{0_{+}}^{\alpha}\left(\int_{0}^{x} k(x, t) \zeta_{\mathrm{k}-1} \mathrm{dt}\right), \mathrm{k}=1,2, \ldots,
$$

From (3.5) and (3.8) it directly follows that:

$$
\left|\zeta(x)-\tilde{\zeta}_{0}(x)\right| \leq \sum_{\mathrm{j}=0}^{\mathrm{n}-1}\left|\epsilon_{\mathrm{j}}\right| \frac{x^{\mathrm{j}}}{\mathrm{j} !} .
$$

Using subsequently the relations (3.6) and (3.9), and the inequality (3.10), we obtain:

$$
\begin{aligned}
\mid \zeta_{1}(x) & -\tilde{\zeta}_{1}(x)|\leq| \zeta_{0}-\tilde{\zeta}_{0}|+| \lambda \mid I_{0_{+}}^{\alpha}\left(\int_{0}^{x}|k(x, t)|\left|\zeta_{0}-\tilde{\zeta}_{0}\right| d t\right) \\
& \leq \sum_{\mathrm{j}=0}^{\mathrm{n}-1}\left|\epsilon_{\mathrm{j}}\right| \frac{x^{\mathrm{j}}}{\mathrm{j} !}+\sum_{\mathrm{j}=0}^{\mathrm{n}-1}\left|\epsilon_{\mathrm{j}}\right||\lambda| \mathrm{N} I_{0_{+}}^{\alpha}\left(\frac{x^{j+1}}{(j+1) !}\right) \\
& =\sum_{\mathrm{j}=0}^{\mathrm{n}-1}\left|\epsilon_{\mathrm{j}}\right|\left(\frac{x^{\mathrm{j}}}{\mathrm{j} !}+\mathrm{N} \frac{x^{j+1}}{\Gamma(j+\alpha+2)}\right) \\
& =\sum_{\mathrm{j}=0}\left|\epsilon_{\mathrm{j}}\right| \sum_{\mathrm{m}=0}^{1}|\lambda|^{\mathrm{m}} \mathrm{N}^{\mathrm{m}} \frac{x^{\mathrm{m}(\alpha+1)+\mathrm{j}}}{\Gamma(m(\alpha+1)+j+1)} .
\end{aligned}
$$

Similarly, we have:

$$
\begin{aligned}
\mid \zeta_{2}(x) & -\tilde{\zeta}_{2}(x)|\leq| \zeta_{0}-\tilde{\zeta}_{0}|+| \lambda \mid I_{0_{+}}^{\alpha}\left(\int_{0}^{x}|k(x, t)|\left|\zeta_{1}-\tilde{\zeta}_{1}\right| d t\right) \\
& \leq \sum_{\mathrm{j}=0}^{\mathrm{n}-1}\left|\epsilon_{\mathrm{j}}\right| \sum_{\mathrm{m}=0}^{1}|\lambda|^{\mathrm{m}} \mathrm{N}^{\mathrm{m}} \frac{x^{\mathrm{m}(\alpha+1)+\mathrm{j}}}{\Gamma(m(\alpha+1)+j+1)}
\end{aligned}
$$

and by induction:

$$
\left|\zeta_{k}(x)-\tilde{\zeta}_{k}(x)\right| \leq \sum_{\mathrm{j}=0}^{\mathrm{n}-1}\left|\epsilon_{\mathrm{j}}\right| \sum_{\mathrm{m}=0}^{1}|\lambda|^{\mathrm{m}} \mathrm{N}^{\mathrm{m}} \frac{x^{\mathrm{m}(\alpha+1)+\mathrm{j}}}{\Gamma(m(\alpha+1)+j+1)} .
$$

Taking the limit of (3.11) as $k \rightarrow \infty$, we obtain:

$$
\begin{gathered}
\left|\zeta_{k}(x)-\tilde{\zeta}(x)\right| \leq \sum_{\mathrm{j}=0}^{\mathrm{n}-1}\left|\epsilon_{\mathrm{j}}\right| \sum_{\mathrm{m}=0}^{1}|\lambda|^{\mathrm{m}} \mathrm{N}^{\mathrm{m}} \frac{x^{\mathrm{m}(\alpha+1)+\mathrm{j}}}{\Gamma(m(\alpha+1)+j+1)} \\
=\sum_{\mathrm{j}=0}^{\mathrm{n}-1}\left|\epsilon_{\mathrm{j}}\right| x^{\mathrm{j}} \mathrm{E}_{\alpha+1, \mathrm{j}+1}\left(|\lambda| \mathrm{N} x^{\alpha+1)},\right.
\end{gathered}
$$

which completes the proof.

It can be deduced from this theorem that small changes in initial conditions cause only small changes of the obtained solution.

\section{Convergence Analysis}

In order to prove that the sequence $\left\{\zeta_{k}(x)\right\}_{k=1}^{\infty}$ is convergent, we construct the series: 


$$
\zeta_{0}(x)+\left[\zeta_{1}(x)-\zeta_{1}(x)\right]+\cdots+\left[\zeta_{k}(x)-\zeta_{k}(x)\right]+\cdots .
$$

Noticing that:

$$
S_{k}(x)=\zeta_{0}(x)+\left[\zeta_{1}(x)-\zeta_{0}(x)\right]+\cdots+\left[\zeta_{k}(x)-\zeta_{k-1}(x)\right]=\zeta_{k}(x),
$$

So, the sequences $\left\{\zeta_{k}(x)\right\}_{k=1}^{\infty}$ will be convergent if all the series, $\sum S_{k}(x)$, are convergent.

Theorem 4.1 Consider the following equation:

$$
\left\{\begin{array}{c}
{ }^{c} D_{0_{+}}^{\alpha} \zeta(x)-\lambda \int_{0}^{x} k(x, t) \zeta(\mathrm{t}) \mathrm{dt}=\mathrm{g}(x), \\
\zeta^{(\mathrm{i})}(0)=\mathrm{c}_{\mathrm{i}}, \mathrm{i}=0,1,2, \ldots, \mathrm{n}-1, \mathrm{n}=[\alpha]+1 .
\end{array}\right.
$$

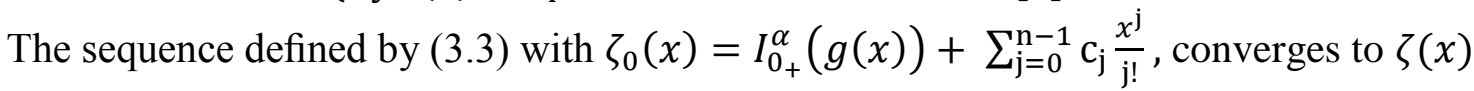
whenever $K 1=\|\zeta 0(x)\|_{\infty}<\infty$ and $K 2=\|k(x, t)\|_{\infty}<\infty$.

Proof. The assumptions imply the following estimations:

$$
\begin{aligned}
\left|\zeta_{1}(x)-\zeta_{0}(x)\right| & \leq|\lambda| I_{0_{+}}^{\alpha}\left(\int_{0}^{x} k(x, t)\left|\zeta_{0}(\mathrm{t})\right| \mathrm{dt}\right) \\
& \leq|\lambda| \mathrm{K}_{1} \mathrm{~K}_{2} I_{0_{+}}^{\alpha}\left(\int_{0}^{x} \mathrm{dt}\right)=K_{1}|\lambda| \mathrm{K}_{2} \frac{x^{\alpha+1}}{\Gamma(\alpha+2)} .
\end{aligned}
$$

Similarly, we have:

$$
\begin{aligned}
\left|\zeta_{2}(x)-\zeta_{1}(x)\right| & \leq|\lambda| I_{0_{+}}^{\alpha}\left(\int_{0}^{x} k(x, t)\left|\zeta_{1}(\mathrm{t})-\zeta_{0}(\mathrm{t})\right| \mathrm{dt}\right) \\
& \leq K_{1}|\lambda|^{2} K_{2}^{2} \frac{x^{2 \alpha+1}}{\Gamma(2 \alpha+2)}
\end{aligned}
$$

Proceeding by induction we obtain:

$$
\left|\zeta_{k}(x)-\zeta_{k-1}(x)\right| \leq K_{1}|\lambda|^{k} K_{2}^{k} \frac{x^{k \alpha+1}}{\Gamma(k \alpha+2)} .
$$

Since the series of:

$$
\sum K_{1}|\lambda|^{k} K_{2}^{k}=K_{1} x E_{\alpha, 2}\left(|\lambda| K_{2} x^{\delta}\right)
$$

is convergent in the whole real line, therefore the series of (4.1) is absolutely convergent, which means that the sequence $\left\{\zeta_{k}(x)\right\}_{k=1}^{\infty}$ is convergent for $x \in[0, X]$.

\section{Test examples}

In order to demonstrate the performance of the present method as a novel solver for integro-differential equations of fractional order, two different problems are selected as test cases. The calculations were done in MAPLE.

Example.5.1 Consider the following integro-differential equation with the following 
initial condition:

$$
\left\{\begin{array}{l}
{ }^{c} D_{0_{+}}^{\alpha} \zeta(x)-\int_{0}^{x}(x-t) \zeta(\mathrm{t}) \mathrm{dt}=x, 0<\alpha \leq 1, \quad 0 \leq x \leq 1, \\
\zeta(0)=0 .
\end{array}\right.
$$

Applying the operator $10_{+}^{\alpha}$, the inverse of the operator ${ }^{\mathrm{c}} D_{0_{+}}^{\alpha}$, to both sides of the above equation yields:

$$
\zeta(x)-I_{0_{+}}^{\alpha}\left(\int_{0}^{x}(x-t) \zeta(\mathrm{t}) \mathrm{dt}\right)=\frac{x^{\alpha+1}}{\Gamma(\alpha+2)}, 0 \leq x \leq 1 .
$$

Its iteration formula reads:

$$
\zeta_{\mathrm{k}}(x)-I_{0_{+}}^{\alpha}\left(\int_{0}^{x}(x-t) \zeta_{\mathrm{k}-1}(\mathrm{t}) \mathrm{dt}=\frac{x^{\alpha+1}}{\Gamma(\alpha+2)}, 0 \leq x \leq 1,\right.
$$

and

$$
\zeta_{0}(x)=\frac{x^{\alpha+1}}{\Gamma(\alpha+2)}
$$

Therefore, we have:

$$
\left\{\begin{array}{c}
\zeta_{1}(x)=\frac{x^{\alpha+1}}{\Gamma(\alpha+2)}+\frac{x^{2 \alpha+3}}{\Gamma(2 \alpha+4)} \\
\zeta_{2}(x)=\frac{x^{\alpha+1}}{\Gamma(\alpha+2)}+\frac{x^{2 \alpha+3}}{\Gamma(2 \alpha+4)}+\frac{x^{3 \alpha+5}}{\Gamma(3 \alpha+6)} \\
\zeta_{3}(x)=\frac{x^{\alpha+1}}{\Gamma(\alpha+2)}+\frac{x^{2 \alpha+3}}{\Gamma(2 \alpha+4)}+\frac{x^{3 \alpha+5}}{\Gamma(3 \alpha+6)}+\frac{x^{4 \alpha+7}}{\Gamma(4 \alpha+8)}
\end{array}\right.
$$

By continuing this way, we obtain:

$$
\zeta_{\mathrm{k}}(x)=\sum_{\mathrm{j}=0}^{\mathrm{k}} \frac{x^{j(\alpha+2)+(\alpha+1)}}{\Gamma(\mathrm{j}(\alpha+2)+(\alpha+2))}
$$

and the exact solution is:

$$
\lim _{k \rightarrow \infty} \zeta_{\mathrm{k}}(x)=\sum_{\mathrm{j}=0}^{\infty} \frac{x^{j(\alpha+2)+(\alpha+1)}}{\Gamma(\mathrm{j}(\alpha+2)+(\alpha+2))}
$$

It is clear that:

$$
\sum_{j=0}^{\infty} \frac{x^{j(\alpha+2)+(\alpha+1)}}{\Gamma(\mathrm{j}(\alpha+2)+(\alpha+2))}=x^{\alpha+1} E_{\alpha+2, \alpha+2}\left(x^{\alpha+2}\right) .
$$

Thus the above sequence is convergent. This confirms that the VIM for integrodifferential equation in (5.1) converges to the exact solution.

Example.5.2 Consider the following integro-differential equation with the following initial condition: 


$$
\left\{\begin{array}{l}
{ }^{c} D_{0_{+}}^{\alpha} \zeta(x)-\int_{0}^{x}(x \sin (t) \zeta(\mathrm{t}) \mathrm{dt})=\mathrm{g}(x), 0<\alpha \leq 1, \\
\zeta(0)=0 .
\end{array}\right.
$$

where:

$$
g(x)=\cos (x)\left(x^{3}+x^{2}-2 x\right)-x \sin (x)+2 x+\frac{2}{\Gamma\left(\frac{5}{2}\right)} x^{\frac{3}{2}}+\frac{1}{\Gamma\left(\frac{3}{2}\right)} x^{\frac{1}{2}} .
$$

Applying the operator $I_{0_{+}}^{\frac{1}{2}}$, the inverse of the operator ${ }^{c} D_{0_{+}}^{\frac{1}{2}}$, to both sides of the above equation yields:

$$
\zeta(x)-I_{0_{+}}^{\frac{1}{2}}\left(\int_{0}^{x} x \sin (t) \zeta(\mathrm{t}) \mathrm{dt}\right)=x^{2}+x, 0 \leq x \leq 1 .
$$

Its iteration formula reads:

$$
\zeta_{\mathrm{k}}(x)-I_{0_{+}}^{\frac{1}{2}}\left(\int_{0}^{x} x \sin (t) \zeta_{\mathrm{k}-1}(\mathrm{t}) \mathrm{dt}\right)=x^{2}+x, 0 \leq x \leq 1
$$

and

$$
\zeta_{0}(x)=x^{2}+x
$$

Then, we obtain $\zeta_{1}, \zeta_{2}, \ldots$ as

$$
\zeta_{k}(x)=x^{2}+x, k=1,2,3, \ldots
$$

Hence $\zeta(x)=\lim _{k \rightarrow \infty} \zeta_{k}(x)=x^{2}+x$ is the exact solution of (5.4).

\section{Conclusions}

In this paper, we applied the VIM to integro-differential equations of fractional order. We have shown that some appropriate conditions guarantee the convergence of the approach. Some examples are presented to illustrate the accuracy of the present method. The numerical cases reveal that obtained solutions are in good agreement with the exact ones. The numerical results show that variational iteration method is very effective and convenient for solving integro-differential equations of fractional order.

\section{Acknowledgments}

We thank the referees for several detailed remarks that led to a substantial improvement in the content as well as the presentation of the paper. The authors also would like to thank Shahrekord University for the financial support through a research grant.

\section{References}

1. Rawashdeh E. A., "Numerical solution of fractional integro-differential equations by 
collocation method", Appl. Math. Comput., 176 (2006) 1-6.

2. Momani S., Noor M. A., "Numerical methods for fourth-order fractional integrodifferential equations", Appl. Math. Comput., 182 (1) (2006) 754-760.

3. Lepik U., "Solving fractional integral equations by the Haar wavelet method", Appl. Math. Comput., 214 (2) (2009) 468-478.

4. Arikoglu A., Ozkol I., "Solution of fractional integro-differential equations by using fractional differential transform method", Chaos, Solitons \& Fractals (2007).

5. Ghasemi M., Fardi M., Khoshsiar Ghaziani R., "A new application of the homotopy analysis method in solving the fractional Volterra's population system", Applications of Mathematics, (2013) In press.

6. Esmaeili Sh., Shamsi M., Dehghan M., "Numerical solution of fractional differential equations via a Volterra integral equation approach", Cent. Eur. J. Phys. (2013) In press.

7. Jafari H., Khalique C. M., Ramezani M., Tajadodi H., "Numerical solution of fractional differential equations by using fractional B-spline", Cent. Eur. J. Phys., (2013) In press.

8. Jafari H., Sayevand K., Tajadodi H., Baleanu D., "Homotopy analysis method for solving Abel differential equation of fractional order", Cent. Eur. J. Phys., (2013) In press.

9. Jafari H., Saeidy M., Baleanu D., "The variational iteration method for solving n-th order fuzzy differential equations", Cent. Eur. J. Phys., 10(1) (2012) 76-85.

10. He J. H., "Variational iteration method-a kind of nonlinear analytical technique: Some examples", Int. J. Nonlinear Mech. 34 (1999) 699-708.

11. He J. H., "A review on some new recently developed nonlinear analytical techniques", Int. J. Nonlinear Sci. Numer, Simul, 1 (2000) 51-70.

12. He J.H., "Non-perturbative methods for strongly nonlinear problems", Dissertation, de-Verlag im Internet GmbH, Berlin (2006).

13. He J. H., Variational iteration method-some relent results and new interpretations", J. Comput. Appl. Math. 207 (2007) 3-17.

14. He J. H., "Variational iteration method for autonomous ordinary differential systems", Appl. Math. Comput. 114 (2000) 115-123.

15. He J. H., Wu X. H., "Construction of solitary solutions and compacton-like solution by Variational iteration method", Chaos Solitons Fractals 29 (2006) 108-113. 
16. Shakeri F., Dehghan M., "Numerical solution of the Klein-Gordon equation via He's variational iteration method", Nonlinear Dynamics 51 (2008) 89-97.

17. Dehghan M., Shakeri F., "Approximate solution of a differential equation arising in astrophysics using the variational iteration method", New Astron. 13 (2008) 53-59.

18. Shakeri F., Dehghan M., "Solution of a model describing biological species living together using the variational iteration method", Math. Comput. Modelling 48 (2008) 2175-2188.

19. Dehghan M., Tatari M., "Identifying an unknown function in a parabolic equation with overspecified data via He's variational iteration method", Chaos Solitons Fractals 36 (2008) 157-166.

20. He J. H., Wu X.H., "Variational iteration method: New development and applications", Comput. Math. Appl. 54 (2007) 881-894.

21. He J. H., "Variational iteration method: Some recent results and new interpretations", Comput. Appl. Math. 207 (2007) 3-17.

22. Kilbas A. A., Srivastava H.H., Trujillo J.J., "Theory and Applications of Fractional Differential Equations", Elsevier Science B.V., Amsterdam (2006).

23. Podlubny I., "Fractional Differential Equations", Academic Press, San Diego (1999). 